(1) colonic irrigation, gastric lavage and hot packs, twice daily; (2) continuous Murphy drips containing potassium acetate, 1 dram to 1 pint of water.

The patient was given by mouth 8 ounces of the following solution every hour, alternating with the same quantity of milk:

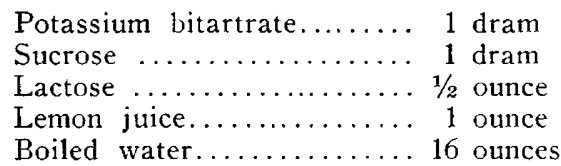

On the first day, November 28, the patient's condition was the same as on admission. On catheterization, 75 c.c. of urine were obtained which, on analysis, showed a very large amount of albumin, a specific gravity of 1.020 , and many coarsely granular and hyaline casts. The patient vomited continuously, and was unable to retain even small amounts of fluid, and refused nourishment. The systolic blood pressure was 136 , and diastolic blood pressure $100 \mathrm{~mm}$. of mercury. The leukocyte count was 11,000 , of which 74 per cent. were polymorphonuclears, and 26 per cent. lymphocytes.

From November 28 to December 2, the patient began to eliminate greater quantities of urine; her condition seemed better, except that she complained of burning pain along the entire gastro-intestinal tract. The restlessness subsided, and she appeared brighter, but continued to refuse food. Gavage and duodenal feeding were resorted to, but because of marked irritability of the esophagus and stomach, had to be discontinued. Hypodermoclyses were then attempted, but soon discontinued because of the excessive pain they gave the patient.

The urine elimination during this period rose from 200 to 700 c.c., and the urine showed albumin, pus and hyaline and granular casts in moderate amounts.

Stomach washings, colonic irrigations, urine and feces, were examined in the laboratory for mercury. The Vogel $^{2}$ test was employed, a positive reaction being indicated by formation of an amalgam of the mercury with gold, in a sealed tube. On the first day the urine and feces gave a positive mercury reaction, on the second day the lavage and feces were positive, and on the third day the urine, feces and gastric contents all responded to the test for mercury.

Noremher 30, a chemical examination was made of the blood, which showed the following interesting figures:

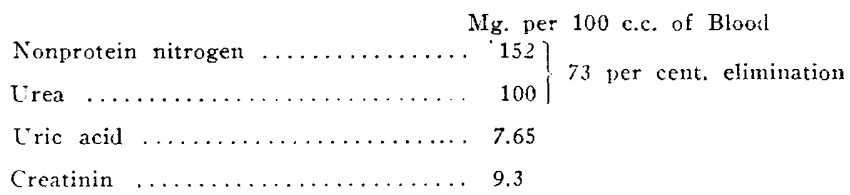

The phenolsulphonephthalein test on this day showed an elimination of 22 per cent. for two hours. These figures for the nonprotein nitrogen and urea are somewhat lower than those reported by Foster, ${ }^{3}$ and Myers and Fine. ${ }^{4}$ No study of chlorid or nitrogen metabolism was attempted, since ingestion of food was made uncertain by vomiting.

From December 2 to December 7 , the patient had severe gastric pains, with continued vomiting. The gastric analysis and string test and roentgenoscopy did not indicate the presence of an ulcer. The patient's general condition was improved, with a urine elimination of 700 c.c. in twenty-four hours, showing a decreased amount of albumin, and few casts. No mercury was found in the excretions, with the exception of the feces, which were positive for one day only (December 4). December 6, the blood was again examined, showing a decrease of nonprotein nitrogen to $32 \mathrm{mg}$. per hundred c.c. of blood, of which the urea nitrogen was 65 per cent., or 21 mg., and an increased phenolsulphonephthalein elimination to 34 per cent.

At the end of this perior the vomiting subsided, the patient took more nourishment, and the feces contained no more mercury. The colon irrigations and lavage were then dis-

2. Vogel, K. M., and Lee, O. I.: Detection of Mercury in the Excretions, Tile Journal A. M. A., Feb. 14, 1914, p. 532.

3. Foster, N. B.: Mercury Nephritis, Arch. Int. Med., April, 1915 p. 754.

. Myers, V. C., and Fine, M. S.: Jour. Biol. Chem., 1915, xv, 391. continued. The blood pressure was lower (systolic 118, diastolic $70 \mathrm{~mm}$. of metcury).

From December 7 to December 11, the patient's mental condition varied considerably. At times she was very irrational, and at other times clear and alert.

December 11, there was a diminution of urine with the appearance of 1.5 per cent. sugar (probably due to excessive ingestion of carbohydrate). The blood analysis, however, showed no hyperglycemia, sugar concentration being 0.64 per cent. There were slight albuminuria, and occasional granular casts.

December 9 , the chemical blood analysis revealed:

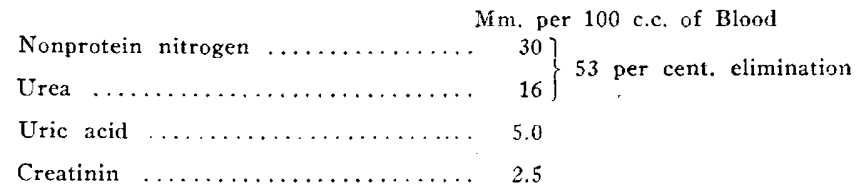

From December 11 to time of discharge, December 31, the patient's general condition steadily improved, with increasing urine elimination ( 900 c.c. daily, and only traces of albumin, and occasional granular and hyaline casts).

SUM MARY

During the first five days, the condition of the patient seemed somewhat improved, after treatment was instituted. Urine elimination varied from 200 to 700 c.c. daily. All the excretions and stomach washings gave positive tests for mercury; the blood showed a marked retention of nonprotein nitrogenous substances and low phenolsulphonephthalein elimination. The next five days of the disease showed a general improvement in the condition of the patient, and practically all excretions showed the absence of mercury; there was increased urine and phenolsulphonephthalein elimination. The nonprotein nitrogen and urea in the blood were markedly decreased. The only other cases reported, those of Foster, and Myers and Fine, showed a greater retention of nonprotein nitrogenous constituents in the blood than our case did. Their cases never showed a decrease of these substances during the progress of the disease, while in our case the nonprotein nitrogenous substances in the blood were markedly decreased as the patient recovered, which was almost coincident with the disappearance of the mercury. The remainder of her stay in the hospital until her discharge was marked by steady improvement in her general condition; she eliminated about the normal amount of urine, which showed only traces of albumin, with occasional hyaline casts.

\section{PURPURA HEMORRHAGICA TREATED WITH NORMAL HORSE SERUM}

Lawrence Schlenker, M.D., St. Louis

This case of purpura hemorrhagica, in a man aged 28 , is instructive in the showing of a prompt response to treatment with normal horse serum.

History.-The patient had mea'sles during childhood. In his eighteenth year he suffered from an illness diagnosed as nephritis, from which he recovered in three weeks. While never robust, he had had no other diseases, and assurance is positive that he was never subject to abnormal bleeding of any kind. There was no knowledge of hemorrhage in any member of his family. The present illness began the middle of December, 1915, with symptoms diagnosed as the grip. The temperature was from 103 to 104 , and there were prostration, cough, and a mucopurulent expectoration. Under his physician's care he improved to some extent, when, January 9, he began to bring up large quantities of thin, bloody sputum. Two days later blood appeared in the urine, the latter soon 
taking on the appearance of pure blood. The following day purpuric spots occurred in the skin, and nasal hemorrhages took place frequently. It is stated that there were no vomiting of blood, no blood in the stools, and no aural hemorrhages. Exsanguination was now taking place so rapidly that the patient's condition was becoming alarming.

Examination.-The patient was admitted to Mount St. Rose Hospital, January 11, in a state of shock. He was extremely pallid and exhausted, dyspneic and in a cold sweat. Pulse and heart sounds were very weak, regular, and 88 to the minute. A dozen or more small purple spots were visible in the skin of the forehead and arms; in the mucous membrane of the soft palate quite a large hemorrhage was present. Both nares were filled with dried blood; the gums and teeth were smeared with blood. The sclera of the left eye contained a fairly large hemorrhage. The urine, as voided, looked much like unmixed venous blood; after standing it separated into two equal layers, a thin, light red upper stratum, and a lower layer thicker and darker. The patient was coughing up more than a pint of bloody, serous fluid in the twenty-four hours, a fluid resembling laked blood somewhat. Examination of the lungs revealed edematous râles over the back and front of both sides of the ehest; there were no signs of consolidation or cavitation. The temperature registered 99 in the morning, and from 103 to 104 through the afternoon and evening. The hemoglobin estimation was 60 per cent., while blood smears were negative, except for a marked paleness of the red cells. No tubercle bacilli were found in repeated sputum examinations, by ordinary smear or by the antiformin method.

Treatment and Result.-The patient was at once given 10 c.c. of normal horse serum, subcutaneously, which dose was repeated daily for the subsequent nine days, making 100 c.c. all told. With the exception of small doses of codein, given the first few days to quiet the nervous symptoms, no other medicine was administered. There was no evidence of serum anaphylaxis at any time. Improvement in sputum, urine and in the general condition was noticeable after the second injection of serum. Blood disappeared entirely from the urine after five injections had been given. The sputum became rapidly less in volume, as well as in blood content. The moist râles in the lungs gradually became less evident.

January 29 there was neither cough nor sputum. The urine was normal in appearance, and negative to tests for albumin and sugar. Temperature was normal throughout the twenty-four hours. The pulse was stronger but still somewhat weak. Examination of the lungs showed entire absence of moist râles.

Strength, weight and color had improved greatly; the patient was up and about the institution. Except for some remaining weakness, which was steadily disappearing, he felt perfectly well.

\section{THE MANAGEMENT OF A CASE OF CUT THROAT}

Ben D. Baird, M.D., Galesblrg, Ill.

I report this case because of its infrequent occurrence, many interesting features, and the various problems it presented which are of interest to the surgeon.

A man, aged 50 years, weighing 94 pounds, and suffering from chronic nephritis, attempted suicide, 3 p. m., Oct. 24 , 1915, by cutting his throat with a paring knife ground down to a fine point. About two hours later he was found lying unconscious in a pool of blood with a gaping wound in his throat. Life was apparently almost extinct because of the loss of blood and his already weakened condition from the chronic nephritis, but an effort was made by the physician to unite the divided trachea temporarily, after which the patient was brought 20 miles to the hospital and was seen by one of my colleagues. Under ether anesthesia the wound was reopened and the divided trachea united and the skin incision closed without drainage. The wound did not involve either of the jugulars or carotids.

On the succeeding morning it was discovered that the patient was unable to swallow, and had been unable to swallow since the infliction of the wound. This was attributed to a loss of motor influences due to a division of the recurrent larnygeal nerve, and was allowed to continue for six days, at which time the patient was in a state of almost complete prostration, owing to the lack of nutrition.

On the sixth day rectal feeding was started. On the eighth day the patient came into my hands, and at that time the wound had broken open. There was a foul smelling discharge coming from it, and at every respiratory effort the air was forced out and sucked in through the wound at the side of the neck, showing that the sutures in the trachea had not held, and he was breathing through the tracheotomy wound instead of the nostrils. A long curved uterine probe was passed into the wound in the neck, and by following the posterior wall in an upward direction the probe appeared in the pharnyx, and I was able to prove that the pharynx had also been opened as well as the trachea divided.

A rubber catheter was then inserted into the wound and passed carefully downward into the stomach, through which the patient was given warm milk, water and brandy, the first nourishment of any kind which had entered his stomach for eight days. The condition of the patient and the condition of the wound were such that it was inadvisalle to close the wound at this time. The problems then were how to build up the patient sufficiently to enable him to withstand the operation which was to follow, and at the same time prevent further irritation of the already diseased kidneys by an anesthetic.

The first problem was met by feeding the patient every eight hours with warm milk, water and brandy through the rubber catheter introduced into the stomach through the esophagotomy. This was continued for nine days.

November 10, under novocain (Crile's nerve blocking method) plus a 2 per cent. cocain solution injected into the tissues as the incision was carried down, gastrostomy was performed according to Witzel's method. The patient suffered practically no discomfort from the operation, and the question of nourishment was considerably simplified, as he was able to pour his own food into the funnel, and his gain in strength was rapid and remarkable. Thirteen days later, again under lacal anesthesia, a tracheotomy was performed below the wound in the trachea, through which the patient was to breathe during and following operation.

Seven days later, when the patient had recovered sufficiently from the immediate effects of his previous operations, he was again returned to the operating room, and with nitrous oxid and oxygen anesthesia given through a soft rubber catheter, which was attached to the tube of the Heidbrink gas machine and introduced into the trachea. I was enabled to enlarge the old self-inflicted wound and found that the action of the muscles acting from below (sternohyoid, omohyoid and thyrohyoid) and above (mylohyoid, geniohyoid and geniohyoglossus) the incision had retracted the divided ends of the trachea and the pharyngeal opening until there was a space $2 \frac{1}{2}$ inches between them. This had converted the transverse incision in the pharynx into a longitudinal one from about 2 to $2 \frac{1}{2}$ inches in length. Granulation tissue had formed around the cut edges of the esophagus, and it was with difficulty dissected out so that the freshened edges might be coapted behind the trachea. I carefully dissected the posterior pharyngeal wall completely loose from its attachments. The freshened edges of the pharynx were brought together by inverting the edges with a very fine plain catgut continuous suture. This line of sutures was then covered with a further row of fine chromatized catgut sutures.

During this portion of the operation the divided end of the thyro-epiglottic ligament was plainly visible projecting downward from the base of the tongue. It was necessary to reconstruct or rather build up a new anterior pharyngeal wall, after which the divided ends of the trachea were freshened, care being taken to prevent blood entering the interior of the larynx where the vocal cords were plainly visible, and with strong No. 3 chromatized catgut interrupted sutures (these heavy sutures were necessary to overcome the action of the muscles), the divided ends of the trachea were brought together, care being taken to make a careful accurate approximation, as there was only the base of the tongue 